\title{
MATEMÁTICA HASTA EL SIGLO XVIII. ¿QUÉ SON LOS NÚMEROS? *
}

J. A. Robles

INSTITUTO DE INVESTIGACTONES FILOS6Ficas Universidad Nacional autónoma de MÉxico

Errores quam minimi in rebus mathematicis non sunt contemnendi...

I. Newton.

Lo que me propongo en este Apéndice es ofrecer al lector un breve panorama de las ideas que acerca de los diferentes tipos de números se manejaban en época de Berkeley señalando, también, el origen de algunas de ellas, así como observaciones posteriores que se hicieron sobre el mismo tema. El objeto que tiene esta presentación es hacer consciente al lector de que si bien Berkeley no fue un creador en el terreno matemático, sin embargo, su posición teórico-crítica ante el panorama que lo rodeaba era una que podemos considerar como de avanzada $y$, ciertamente, en los casos en los que Berkeley no tiene claridad acerca de una posición matemática determinada, veremos que los matemáticos de la época tampoco son nada claros en muchos aspectos importantes de su disciplina.

Según la presentación general que hice en el texto principal de este capítulo, Berkeley, en sus Philosophical Commentaries, nos ofrece una versión nominalista de la aritmética —de la matemática en general, si excluimos a la geometría. Una posición así, a pesar de su tono antimetafísico, tiene que dar cuenta de qué son los números; 0 , mejor dicho, debe dar cuenta de cuál sea el significado de nuestras expresiones matemáticas. Berkeley intenta dar esta cuenta, según lo mostré en el texto principal, en términos relacionales. Para ser preciso, hay que señalar que la posición de Berkeley es una pragmático-instrumentalista, plenamente coherente con su posición nominalista acerca de las expresiones matemáticas. El presente texto le ofrecerá al lector propuestas que se oponen claramente a la de Berkeley; en su mayor parte son realistas, concediéndoles ser a los relata de las expresiones numéricas. Pero el lector podrá apreciar, también, que las propuestas teóricas de los mate-

- Apéndice al capítulo II, "Aritmética y álgebra" del libro en preparación Las ideas matemáticas de George Berkeley, obispo de Cloyne. Aprovecho la oportunidad para agradecer a $M$. Beuchot su lectura detenida de una versión anterior $y$ sus observaciones acerca de las versiones españolas de los textos latinos que figuran en el presente escrito. A. Herrera me auxilió también con los textos latinos, gracias. 
máticos, acerca de los números, son oscuras en muchas ocasiones o claramente contradictorias en otras. Espero que la presentación de estos textos haga consciente al lector de la claridad y agudeza que caracterizaron al obispo Berkeley en la mayoría de sus trabajos teóricos.

\section{Introducción}

La situación en matemáticas, tal como se presenta al inicio del siglo xviII, da la impresión de ser un bazar desordenado en el que se ofrecen diversas mercancías que no parecen tener demasiado en común: hay números enteros (no negativos) que son, con mucho, los que han adquirido un mayor arraigo entre los matemáticos europeos. Junto a éstos se encuentran los racionales (números fraccionarios) positivos que tampoco tienen, ya, nada que temer. Sin embargo, para llegar a alcanzar un sistema numérico decente y adecuado aún faltan varios elementos, acerca de los que hubo bastantes desacuerdos. Comenzaré por considerar los números irracionales (positivos).

\section{Nümeros irracionales}

Morris Kline, en su excelente historia de la matemática, nos dice:

Más o menos por el 1500, el cero se aceptó como un número y los números irracionales se usaron con mayor libertad. Pacioli, el matemático alemán Michael Stifel (1486(?)-1567), el ingeniero militar Simón Stevin (1548-1620) y Cardán, usaron los números irracionales conforme a la tradición de hindúes y árabes e introdujeron más y más tipos de aquéllos. ${ }^{1}$

El lector recordará que un número irracional es un número como $\sqrt{2}$ que no puede expresarse en términos de una razón entre enteros ${ }^{2}$ y cuya representación se hace en términos de una sucesión infinita de

1 En [9], p. 251. Pacioli, a quien Kline menciona en la cita, fue Fra Luca Pacioli (ca.1445-ca.1514), monje italiano que fuera discfpulo de Pierro della Francesca y amigo y maestro de Leonardo. Por lo que respecta al cero, puedo añadir a lo que nos dice Kline, que Fibonacci lo usa en su Liber Abacci (cf. p. 158 y n. 7), publicado en 1202. El cero lo toma Fibonacci de los textos árabes que conoció gracias a sus viajes por los países del Islam. La palabra árabe para cero, as-sifr, originó, en diversos idiomas, las palabras equivalentes a "cero" y "cifra" ("chiffre" en francés, "Ziffer" en alemán, "Zero" y "cipher" en inglés) y estas mismas palabras en castellano. Cf. [18], p. 2 y p. 2, n. 1. "Cifra" y "cero" se llegaron a usar indistintamente aún hasta el siglo xvII por Napier, por ejemplo, según lo señala Struik: [18], p. 12, n. 1.

2 Para una versión más detallada del surgimiento del problema de los irracionales entre los pitagóricos, cf. el Apéndice $A$ del Cap. III. 
decimales, sin que en la misma haya periodicidad alguna. Un comienzo de representación de la $\sqrt{2}$ anterior, es:

$$
\sqrt{2}=1.41421356237 \ldots
$$

Justamente fue el descubrimiento de los números irracionales lo que provocó la primera gran crisis matemático-filosófica y lo que hizo que los griegos, al no poder manejar los irracionales como números, le dedicaran tanta atención a la geometría y muy poca a la aritmética. Pero esta historia la narro en el siguiente capítulo por lo que, ahora, será conveniente dar un salto de más de veinte siglos y llegar, así, hasta el siglo Xvi, cuando Michael Stifel nos dice lo siguiente acerca de los irracionales:

Puesto que al demostrar figuras geométricas si nos fallan los números racionales, los números irracionales toman su lugar $\mathrm{y}$ demuestran exactamente aquello que no podian demostrar los racionales... nos vemos llevados y obligados a afirmar que verdaderamente son números; obligados, esto es, por los resultados que se siguen de su uso - resultados que, según percibimos, son reales, ciertos y constantes. Por otra parte, otras consideraciones nos impulsan a negar que de alguna manera sean números los números irracionales. A saber, cuando intentamos sujetarlos a numeración [forma decimal]... encontramos que huyen perpetuamente de tal manera que ninguno de ellos puede aprenderse con precisión y en sí mismo... Ahora bien, aquello que es de tal naturaleza que carece de precisión, no puede denominarse un número verdadero... Por tanto, así como un número infinito no es un número, así un número irracional no es un número verdadero, sino que se encuentra oculto tras una especie de nube de infinitud. ${ }^{8}$

Con algunos autores, sin embargo, la situación había cambiado hacia finales del siglo xvI y principios del xvir. Simón Stevin y John Wallis (1616-1703) aceptaron los irracionales como números en sentido pleno; el primero encontró aproximaciones cada vez más precisas a aquéllos por medio de racionales. ${ }^{4}$

\section{Números negativos}

Cito una vez más a Kline:

Por lo que toca a los números negativos, aun cuando se habían conocido en Europa a través de los textos árabes, la mayoría de los

3 Citado en [9], p. 251; pero véase, también [10], p. 114.

I Cf. [9], p. 252 y [10], p. 114. 
matemáticos de los siglos dieciséis y diecisiete no los aceptaron como números o, si lo hicieron, no los aceptaron como raf́ces de ecuaciones. ${ }^{5}$

Paso ahora a presentar una brevísima narración de los avatares de estos números.

Los hindúes los introducen para representar deudas. El primer uso conocido de los negativos se tiene por Brahmagupta (598-?) quien los presenta en, aproximadamente, el año 628.

Un importante matemático hindú, posterior a Brahmagupta, Brahmin Bhaskara (1114-ca.1185), señaló que la raíz cuadrada de un número positivo es doble: positiva y negativa. Por otra parte, y adelantándome a un tema que tocaré más adelante, señalo que Bhaskara tuvo en cuenta, en algún momento, la raíz cuadrada de un número negativo, pero dice que no hay tal raíz cuadrada, porque un número negativo no es un cuadrado. $^{8}$

A diferencia de los hindúes, los árabes dieron un paso atrás y rechazaron los números negativos.

En 1202, Leonardo de Pisa, Fibonacci (ca. 1170-1250), escribe su Liber Abaci (o Libro del ábaco, revisado en 1228; este libro circuló profusamente en forma de mànuscrito, pero no fue publicado sino hasta 1857: Scritti di Leonardo Pisano) ${ }^{\gamma}$ y, en él, su autor sigue la costumbre árabe de no prestar atención a los negativos; sin embargo, en 1225 escribe un tratado, Flos, en donde interpreta una raf́z negativa, en un problema financiero, como significando una pérdida en lugar de una ganancia. ${ }^{8}$

Aún habrían de pasar varios siglos para que los números negativos fueran plenamente reconocidos como tales. En el siglo xvi Gerolamo Cardano, Hieronymus Cardanus o, directamente, Jerónimo Cardán (1501-1576), en 1545, denominó a los números positivos "numeri ueri" $\mathrm{y}$ "numeri ficti" a los negativos.

Michael Stifel en su Arithmetica Integra, denominó "absurdos" a los números negativos: "Figuntur numeri minores nihilo sunt $0-3$ " y, más adelante, habla del cero como "quod mediat inter numeros veros et numeros absurdos".9

5 En [9], p. 252.

6 En [5], p. 93, Cajori nos dice que "los hindúes fueron los primeros en reconocer la existencia de cantidades absolutamente negativas. Ellos pusieron de manifiesto la diferencia entre cantidades positivas y negativas ligando con unas la idea de 'posesión' y la idea de 'deuda' con las otras". Para conocer la posición de Bhaskara y otros datos interesantes sobre números complejos, cf. [17], II, p. 261 y ss.; además, véase la cita de Bhaskara en p. 164.

7 El libro lo publica B. Boncompagni, Tipografia delle scienze matematiche e fisiche, Roma; 2 vols.; pp. 459. Citado en [18], p. 2.

8 En [17], p. 258; acerca de esto Fibonacci sigue el uso de los hindúes; cf. n. 6.

9 "Un ejemplo de un número menor que nada es $0-3$ "; y del cero nos dice que 
Pasemos ahora al siglo xvir y detengámonos en La Géométrie (1637) de Descartes (1596-1650). En esta obra, su autor nos dice:

Mais souvent il arrive, que quelques unes de ces racines son fausses ou moindres que rien... ${ }^{10}$

y, la razón de la falsedad de tales raíces es similar a la que apunta Stifel: ¿es posible que haya números menores que nada $(=0)$ ?

Ya bien entrado el siglo xvIII, Jean Le Rond D'Alembert (1717-1783), en la Encyclopédie, en el artículo "Négative", escribe:

Ainsi les quantités négatives indiquent réellment dans le calcul des quantités positives, mais qu'on a supposées dans une fausse position. Le signe - que l'on trouve avant une quantité sert à redresser \& à corriger une erreur que l'on a faite dans l'hypothèse...11

Finalmente, deseo destacar una opinión de un autor del siglo xviIr que parece reflejar fielmente el malestar que muchos matemáticos sen-

es "lo que media entre los números verdaderos y los números absurdos". Citado en [17], II, p. 260 y n. 6.

10 "Pero, con frecuencia sucede que algunas de estas raíces son falsas o menores que nada..."; en [6], p. 158. Vale la pena mencionar aquí que John Wallis mantuvo la misma posición que hemos visto que sostenían Stifel y Descartes con relación a los negativos; sin embargo, mantenía que tales números eran mayores que lo infinito, pero no menores que cero. Cf. [9], p. 253.

11 En [7], vol. XI. La observación de D'Alembert es interesante y el ejemplo que ofrece es sugerente. Tal ejemplo, lo presenta D'Alembert en forma del siguiente problema: ¿Cuánto es preciso sumarle a 100 para obtener 50? Esto es, ¿cuál es el valor de $x$ en la ecuación

$$
100+x=50 ?
$$

D'Alembert señala que el resultado,

$$
x=-50 \text {, }
$$

muestra que nuestra formulación del problema estaba equivocada, ya que lo que debería haberse pedido es lo que habría que restarle a 100 para obtener 50 ; la raiz negativa de la ecuación nos muestra que hay algo que modificar en el punto de partida: un error ahi conduce a una respuesta negativa. No será sino hasta más tarde (en el siglo xux) que podrán considerarse ciertos problemas con abstracción de todo contenido y que los números negativos se aceptarán con plenos derechos dentro de la ciudadania de los números.

Pero en todo esto parece haber habido una omisión; mis disculpas al lector por haber dejado la cita sin traducir. Lo hago de inmediato: "De esta manera, las cantidades negativas indican realmente, en el cálculo, cantidades positivas a las que se ha supuesto en una posición falsa. El signo - que se encuentra delante de una cantidad, sirve para enderezar y corregir un error que se ha cometido en la hipótesis..."

Una muestra más del ingenio de D'Alembert nos permitirá tener una idea mejor de los problemas que fueron capaces de provocar las cantidades negativas en las mentes de los matemáticos. El volumen de la Encyclopédie donde figura el artículo "Négative" de D'Alembert que aquí nos ocupa (volumen XI), se publicó en 1765; un 
tían con respecto a los números negativos. En su libro Dissertation on the Use of the Negative Sign in Algebra (1759), el Barón Francis Masères (1731-1824), matemático inglés, escribió acerca de las raíces negativas que

.. hasta donde soy capaz de juzgar, tan sólo sirven para crear perplejidades en toda la doctrina de las ecuaciones y para convertir en oscuras y. misteriosas cosas que, por su misma naturaleza, son excesivamente claras y simples...

Sería de desear, pues, que las raíces negativas nunca hubiesen sido admitidas en el álgebra o, que una vez más, se descartasen de ella. ${ }^{12}$

\section{Números imaginarios}

Berkeley, además de lo que ya he señalado con respecto a su perplejidad en relación a la matemática de su época, encuentra otro motivo de asombro en la misma, que expresa con las siguientes palabras:

Mem: Raices imaginarias desentrañar ese Misterio. ${ }^{13}$

Esto lo escribe entre 1707-8; no es sino hasta 1731, en su libro en forma de diálogo, Alciphron, donde vuelve a retomar el tema y propone una manera de eliminar el "misterio":

que la verdadera finalidad del discurso... no es tan sólo, de manera principal o siempre, impartir o adquirir ideas sino, más bien,

siglo antes, Antoine Arnauld (1612-1694), teólogo, matemático y amigo cercano de Pascal, puso en cuestión la verdad del enunciado de proporcionalidad siguiente:

$$
(-1): 1:: 1:(-1)
$$

pues, según decía, dado que $(-1)$ es menor que $(+1)$, ¿cómo puede ser algo menor a algo mayor tal como lo mayor es a lo menor? cit. en [9], p. 252.

A la queja de Arnauld, así como a las de Stifel, Descartes y otros, responde D'Alembert de la siguiente manera:

Il faut avouer qu'il n'est pas facile de fixer l'idée des quantités négatives, \& que quelques habiles gens même contribué à l'embrouiller par les notions peu exactes qu'ils eu ont données. Dire que la quantité négative est au-dessous du rien, c'est avancer une chose qui ne se peut pas concevoir. Ceux qui pretendent que 1 n'est pas comparable à -1 , \& que le rapport entre 1 \& -1 est différent $d u$ rapport entre -1 \& 1 , sont dans une double erreur: $1^{9}$ parce qu'on divise tous les jours dans les opérations algébriques, 1 par - 1: 2o l'égalité du produit de -1 par -1 \& de +1 par +1 , fait voir que 1 est a -1 comme -1 a 1 .

12 Citado en [10], p. 119.

13 El pasaje es PC 764; en [2], p. 271. 
una especie de naturaleza operante, activa, que tiende hacia un bien concebido el cual puede obtenerse, en ocasiones... incluso cuando no hay posibilidad de ofrecer o de exhibir una idea así... Por ejemplo, la marca algebráica que denota la raíz de un cuadrado negativo, se usa en operaciones logísticas aun cuando sea imposible formar una idea de cualquier cantidad semejante. ${ }^{14}$

La perplejidad de que da muestra Berkeley no es algo único de él. La misma la sintieron agudos matemáticos anteriores a Berkeley así como otras mentes lúcidas posteriores, hasta llegar a obtener una manera de representar los números imaginarios gracias al ingenio de Argand, Wessel y, nada menos, que el gran Gauss. Pero, mejor será hacer un recorrido de esta historia, más pausado y desde un principio.

Bueno, ¿pero qué son estos números imaginarios de los que hasta aquí he hablado sin dar demasiados datos acerca de ellos? El mismo apelativo de "imaginarios" ¿no los excluye ya del ámbito de los que son realmente números?

Una primera observación acerca de nomenclatura. Los pitagóricos consideraban que el mundo podía entenderse y explicarse a través de los números enteros (positivos) y de relaciones (razones) entre ellos. La aparición de números (?) que no podían expresarse como enteros o razones entre éstos, produjo la primera gran crisis teórica en la visión matemática del mundo. Los extraños números de los que tuvieron conocimiento los pitagóricos recibieron el reprobable nombre de "irracionales", justamente porque no se apegaban a los cánones de racionalidad que ellos exigian de cualquier entidad que fuese un número. ${ }^{15}$ Ahora

14 En Alcph., VII, 14; [1], p. 307.

15 Al lector ciertamente le extrañará encontrar afirmaciones como las que ha venido leyendo a lo largo del presente escrito. Nuestra familiaridad con algunos de los números de los que aquí he hablado y otros de los que hablaré más adelante, nos crea frenos para entender cómo fue posible mirar tales números con extrañeza. Sin embargo, los mismos pasaron a formar parte de nuestra cultura (no de nuestra naturaleza) en algún momento y las citas que hasta ahora he presentado y otras más que iré presentando a lo largo de este Apéndice, harán consciente al lector de los problemas y dificultades que lleva aparejado consigo aceptar algunos cambios o ampliaciones en nuestras estructuras de creencias.

Esta situación no concluyó en épocas pasadas; a finales del siglo pasado Georg Ferdinand Ludwig Phillipp Cantor (1845-1918) introduce los números cardinales transfinitos y, acerca de esto, nos dice Russell: "Cuando por primera vez se introducen los números infinitos a la gente, ésta es capaz de negarles el nombre de números, pues la conducta de éstos es tan diferente de la de los números finitos que parece un mal empleo premeditado de los términos el llamarlos números..." [16], p. 203. Pero véase todo el capítulo VII: "La teoría positiva del infinito", recogido en [15], Cap. 5, pp. 83-105.

Nuestra resistencia al cambio la registró también Berkeley, de manera aguda, señalando que: "Prejuicios viejos y arraigados se convierten con frecuencia en principios y las proposiciones que en algún momento obtienen la fuerza y el crédito de un 
bien, la historia prosiguió y, felizmente, los racionales y los irracionales (no sin problemas, según hemos visto) lograron complementarse de manera adecuada y, conjuntamente, formaron el sistema de números reales. Fuera del mismo quedaron esos extraños engendros usados conscientemnete por primera vez por Cardán, a saber, los imaginarios. Carl Friedrich Gauss (1777-1855), consciente de la fuerza que puede tener una denominación para impedirnos hacer evaluaciones adecuadas de las cosas nombradas inadecuadamente, cambió ese nombre de "imaginarios" por el de "complejos" para designar a los números que aquí nos ocupan. Pero esto no fue sino hasta finales del siglo xviII o principios del XIX, por lo que dejamos aquí esta observación y nos volvemos al siglo xvi.

Una vez apuntado el aspecto terminológico, volvamos con mi pregunta inicial: ¿qué son esos "números" imaginarios? Para responder esta pregunta, le recordamos al lector lo que es la raiz cuadrada de un número cualquiera, $a$, por ejemplo. Lo que acerca de esto puedo decir es que:

$$
\sqrt{a}=b \text { si y sólo si } b^{2}=b b=a ;
$$

eșto es, la raíz cuadrada de un número $a$ es un número $b$ si y sólo si $b$ multiplicado por sí mismo (esto es, $b$ elevado al cuadrado o $b^{2}$ ) es igual a $a$. Alguno de los casos que el lector recuerda son, por ejemplo:

$$
\sqrt{4}=2, \quad \text { porque } 2 \cdot 2=4
$$

y, también

$$
\sqrt{4}=(-2) \text { porque }(-2) \cdot(-2)=4 \text {. }
$$

Comprimiendo un poco la notación, y cambiando el ejemplo, se puede expresar lo anterior de la siguiente manera:

$$
\sqrt{9}= \pm 3
$$

Lo que es importante que el lector tenga en cuenta es que el número al que se le extrae la raíz cuadrada es siempre positivo. Pues, por la ley de los signos, ningún número (real), multiplicado por si mismo, dará como resultado un número negativo. $\mathrm{Y}$ es justamente por razones de signo por la que surgen los números imaginarios, como algo extraño y diferente de los números reales. Un ejemplo de los primeros, sería, principio, no se cree tan sólo que ellas sino, igualmente, todo lo que de las mismas se deduzca, se encuentra exento de cualquier examen. $\mathrm{Y}$ no hay absurdo, por grande que sea que, por este medio, no esté dispuesta a tragarlo la mente humana"; en $P C H$, I, 124. [4] II, pp. 98-9. 


$$
\sqrt{(-4)}
$$

y este número (?) no es ni puede ser un número real, pues según nuestra caracterización $(\alpha)$, no hay ningún número real, $b$, que multiplicado por si mismo, nos dé como resultado $(-4)$. Así pues, $\sqrt{(-4)}$ representa un tipo diferente de número, el tipo de los números imaginarios. Sin embargo, la separación entre imaginarios y reales se puede atenuar de la siguiente forma:

$$
\sqrt{(-4)}=\sqrt{4 \cdot(-1)}=\sqrt{2^{2} \cdot(-1)}=2 \sqrt{(-1)}=2 \mathrm{i}
$$

y lo que abtenemos es un número formado por una parte real, "2", y por la unidad imaginaria, " $\mathrm{i}$ ", la que tiene la siguiente propiedad:

$$
\mathrm{i}^{2}=(-1) \cdot{ }^{16}
$$

16 Vale la pena dar aquí una versión de la representación geométrica de los números complejos y hacer un par de aclaraciones terminológicas. Según lo que he señalado en el texto, un número imaginario está caracterizado por ser una expresión de la forma

(i) $Y i$,

donde la " $Y$ " representa un número real $e$ " $i$ " representa la unidad imaginaria. Sin embargo, nos enfrentamos a expresiones más complicadas (cf. p. 165) de la forma

$$
\mathrm{X} \pm \mathrm{Yi}
$$

donde " $Y$ " y " $X$ " son números reales. En caso de que $X=0$, en (ii) nos quedaríamos con la parte puramente imaginaria, que es (i); en caso de que fuera $Y=0$, nos quedaríamos, en (ii), con la parte puramente real, a saber,

\section{$X$.}

La complejidad de los números complejos la muestra (ii) con toda claridad, donde figuran una parte puramente real y una imaginaria reunidas por un símbolo de operación $(+0-)$.

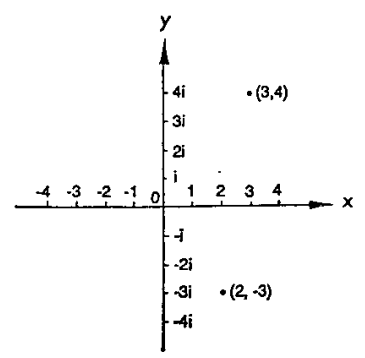

Con respecto a la representación, ésta se puede llevar a cabo, de manera simple, mediante el trazo de dos ejes coordenados, $x, y$, tales que uno de ellos $(x)$ sea el eje real y el otro $(y)$ el eje imaginario - tal como se ve en la figura-, donde, en sus ejes respectivos, se encuentran perfectamente ordenados, de manera lineal, los números puramente reales y los puramente imaginarios. Ahora podemos representar, 
No quiero seguir abusando de la paciencia del lector; pero, decir lo anterior, me pareció necesario para hacer inteligible lo que sigue. Continúo ahora mi historia interrumpida.

Conforme a la historia de la matemática que nos narra D. E. Smith, ${ }^{17}$ el primer rastro de la raíz cuadrada de un número negativo, en obras existentes, se encuentra en la Stereometria de Herón de Alejandría (50-?); en su Arithmetica, Diofanto (ca. 275) también se enfrenta a tales números. Dando ahora un salto de varios siglos, lleguemos a Bhaskara, en la India del siglo xII, quien nos dice:

El cuadrado de una cantidad afirmativa [positiva] o de una negativa, es afirmativo y la raíz cuadrada de una cantidad afirmativa es doble, positiva y negativa. No hay raíz cuadrada de una cantidad negativa, pues no es un cuadrado. ${ }^{18}$

En el siglo $x v$ encontramos reacciones de rechazo a problemas en los que figuran raíces cuadradas de números negativos. No es sino hasta el siglo xvi, con Jerónimo Cardán, que se usan los imaginarios para resolver ecuaciones, pero aún con mala conciencia, según veremos en un momento. Cardán, en 1545, publica en Nuremberg su libro Ars Magna en el que, fuera del caso que considero a continuación, intenta dejar de lado los imaginarios cuando éstos se le presentan. ${ }^{10}$ El caso que Cardán considera es el siguiente: cómo dividir 10 en dos partes tales que su producto sea (30 o) 40 . Si consideramos el caso en el que el producto sea 40 y usamos notación contemporánea, el problema se plantea en términos del siguiente sistema de ecuaciones con dos incógnitas:

$$
x+y=10
$$

$$
x y=40
$$

en el plano, un número complejo cualquiera por coordenadas (iii) $(x, y)$ tales que $\boldsymbol{x}$ representa la parte real e $y$ la imaginaria. Si $x=0$, (iii) representa un número puramente imaginario en el eje $y$ y si, en cambio, $y=0$, (iii) representa un número real en el eje $x$. Si $x=0=y$, (iii) representa el origen 0 que es común para reales e imaginarios. Lo que es interesante señalar aquí es que los números complejos no se encuentran ordenados en línea (como sucede con los reales o con los imaginarios puros), sino en el plano.

En el caso de que el lector tenga deseos de enterarse de más propiedades, usos, etc., de los números complejos, una primera aproximación para verlos en un contexto más amplio, puede serlo muy bien [14], cap. 15, o bien el grato libro de Gamow [8], pp. 32-9.

I7 [17], II, p. 261.

18 Idem.

19 Cf. [18], p. 63. 
despejando $x$ en (1), tenemos:

$$
x=10-y
$$

y sustituyendo $x$ en (2), nos da:

$$
(10-y) y=40 .
$$

Efectuando las operaciones, ordenando y multiplicando ambos lados de (3) por $(-1)$, obtenemos:

$$
y^{2}-10 y=(-40)
$$

completamos el cuadrado a la izquierda de la igualdad (4) -esto es, sumamos 25 a ambos lados de la ecuación-y obtenemos:

$$
y^{2}-10 y+25=(-15) \text {, }
$$

lo que es igual a:

$$
(y-5)^{2}=(-15)
$$

Y si ahora extraemos raíz cuadrada a ambos lados de la igualdad (6), nos queda:

$$
y-5=\sqrt{(-15)}
$$

lo que, finalmente, da como valor de $y$ :

$$
y=5 \pm \sqrt{(-15)},
$$

y esto permite resolver el sistema de ecuaciones (1) y (2).

Lo que me interesa señalar aquí es que inmediatamente después de la formulación del problema, Cardán mismo señala: “...manifestum est quod casus seu quæstio est impossibilis..." ("...es evidente que este caso o cuestión es imposible..."); y, al concluir su demostración (¿que debería ser imposible puesto que surge de una cuestión imposible según nos dijo?), añade: “...et hucusquæ (sic) progreditur Arithmetica subtilitas, cuius hoc extremum ut dixi, adeo est subtile, ut sit inutile ("... así progresa la sutileza aritmética cuyo fin, según he dicho, es tan sutil como inútil").20

20 Existe reproducción fotográfica de una página del texto de Cardán donde figuran los pasajes citados, en [18], p. 68; Struik proporciona, también, una traducción del texto de Cardán en Ibid., pp. 67 y 69. 
Albert Girard (1595-1632), en su libro L'Invention nouvelle en l'algèbre (Amsterdam, 1629) sitúa los números negativos a la par de los positivos y da ambas raíces de una ecuación cuadrática, incluso cuando ambas son negativas; sin embargo, al obtener un resultado imaginario como solución de la ecuación

$$
x^{2}=6 x-25,
$$

llama "inexplicable" a tal resultado, ${ }^{21}$ a saber,

$$
x=3 \pm \sqrt{(-16)}
$$

A pesar de esto, el mismo Girard encontró necesario reconocer las ráces complejas (o imaginarias), a fin de establecer la ley acerca del número de raíces de una ecuación. (El teorema fundamental del álgebra que dice que una ecuación de grado $n$ tiene, exactamente, $n$ rafces, sean éstas reales o complejas; el teorema lo demuestra definitivamente Gauss a finales del siglo xvinI.) Girard, acerca de esto, nos dice:

On pourroit dire à quoy sert ces solutions qui sont impossibles, $j e$ respond poir trois choses, pour la certitude de la reigle generale, et qu'il ny a point d'autre solutions, et pour son utilité..2

Hasta ahora hemos encontrado estados de perplejidad ante los números imaginarios; esta situación proseguirá aún por algún tiempo. Sin embargo, antes de ofrecerle al lector más ejemplos de esto, le presento el pasaje de La Géométrie de Descartes donde los números que ahora nos ocupan, y que han sido tachados de imposibles, reciben el apelativo de imaginarios. He aquí el pasaje:

Au reste tant les vrayes racines que les fausses ne sont pas toujours réeles; mais quelquefois seulement imaginaires; c'est a dire qu'on peut bien toujours en imaginer autant que iay dit en chasque Equation; mais qu'il n'y a quelquefois aucune quantité, qui corresponde a celles qu'on imagine. ${ }^{23}$

21 Cf. [17], II, p. 262.

22 Este texto, traducido con cierta liberalidad, dice: "Se podría preguntar para qué sirven estas soluciones que son imposibles; la respuesta que doy es que sirven para tres cosas: para la certeza de la regla general, porque no hay ninguna otra solución y por su utilidad." Citado en [17], II, p. 262, n. 8.

23 El texto se encuentra en [6], p. 174; traducido, dice:

Por lo demás, tanto las rafces verdaderas como las falsas, no son siempre reales. sino, en ocasiones, tan sólo imaginarias; es decir, que muy bien se puede siempre, en cada ecuación, imaginar todo lo que yo he dicho pero que, en ocasiones, no hay ninguna cantidad que corresponda con lo que uno imagina. 
Lo que Descartes quiere subrayar con esta caracterización, es que las cantidades con las que aquí trata son puramente imaginarias; esto es, como lo señala al final de la cita, no hay ninguna cantidad que corresponda con lo que imaginamos. Parece que lo que él pretende distinguir son aquellas cantidades que tienen un tipo de existencia independiente del acto de pensar (imaginar) de un sujeto y aquellas que tienen una existencia dependiente. Estas últimas son las cantidades o números imaginarios. Pero, tiene algún sentido hablar de cantidades puramente imaginarias? No lo tiene si se mantiene una tesis realista acerca de las entidades matemáticas; por lo que si las cantidades en las que piensa Descartes han de ser entidades independientes, objetivas y, por esto, si lo que figura en nuestra imaginación tiene algo objetivo que le corresponda, en el caso de las cantidades imaginarias no hay cantidades externas que imaginar; esto es, las cantidades imaginarias son tales ique no son ellas mismas imaginables! Dejamos, aquí, este problema abierto.

Si ahora nos movemos hasta principios del siglo XVIII, encontramos la más notable caracterización de los números imaginarios, debida a la imaginación de Gottfried Wilhelm Leibniz (1646-1716):

Itaque [Divina Mens] elegans \& mirabile effugium reperit in illo Analyseos miraculo, idealis mundi monstro, pene inter Ens \& nonEns Amphibio, quod radicem imaginariam appellamus. ${ }^{24}$

Dejo al lector gozando de la notable descripción leibniziana y prosigamos nuestro viaje por el tiempo. Más avanzados en el siglo xviII, Leonhardt Euler (1707-1783) nos dice en su Vollständige Anleitung zur Algebra (publicada en ruso en 1768-9 y en alemán en 1770):

Puesto que todos los números concebibles son o bien mayores que cero, menores que cero o iguales a cero es claro, entonces, que las raíces cuadradas de los números negativos no pueden incluirse entre los números posibles [los números reales]. En consecuencia, debemos decir que éstos son números imposibles. $\mathrm{Y}$ esta circunstancia nos lleva al concepto de tales números que, por su naturaleza, son imposibles y, de ordinario, se les denomina imaginarios o de la fantasía, porque existen sólo en la imaginación..$^{25}$

En general, todas las citas que hasta ahora hemos visto son objetables en tanto que, en unos casos, se habla de imposibilidades útiles (la idea

24 De esta manera [la Mente Divina] encontró una salida elegante y sublime en ese milagro del análisis, en ese prodigio del mundo ideal, casi anfibio entre el ser y el no ser, que denominamos rafz imaginaria. En [13], p. 378.

25 Citado en [9], p. 594. 
tras esta descripción podría ser que se trata de entidades (?) cuya existencia es imposible pero que los enunciados que aluden -que pretenden aludir - a ellas son útiles en la computación; ${ }^{26}$ quizás hubo otras motivaciones más oscuras tras las tesis que se expresaban acerca de los imaginarios. Berkeley, quiero subrayarlo una vez más, no se dejó llevar por los manejos lingǘsticos fáciles y no fue culpable de enunciados tan obviamente paradójicos como los que hemos visto expresados por los matemáticos; sobre esto diré más en un momento), en tanto que, en la última cita, Euler nos habla de imposibilidades iposibles a la imaginación! lo que se acerca mucho a la caracterización cartesiana que vimos páginas atrás.

Las citas que hasta aquí he presentado muestran, sin lugar a dudas, las enormes dificultades conceptuales a las que se enfrentaron los matemáticos para dar cuenta de sus hallazgos. La lucha con los conceptos y las contradicciones no puede ser más evidente. A pesar de todo esto, la matemática siguió avanzando regida, básicamente, por el criterio ipragmático! de utilidad. Pero, por esta razón, y para el caso especifico de los números imaginarios, ¿no debería haberles sido claro a los matemáticos que tenían que habérselas con algo --en caso de tener que postular entidades matemáticas_?, no tan sólo posible sino objetivo.

Berkeley pudo haberles enseñado mucho a los matemáticos de su época mediante sus críticas y su nominalismo en matemáticas. Conforme a los criterios de Berkeley, en matemáticas no hay problema alguno acerca de si existen o no entidades matemáticas. La pregunta básica, según su manera de ver las cosas, es ¿son útiles tales o cuales expresiones matemáticas? Si lo son, con eso tienen asegurado su pase al mundo de los sistemas matemáticos; en caso contrario, Berkeley pide que se las destierre de tal mundo. (Sin embargo, a esto puede replicarse que si el criterio de significado que propone Berkeley es el de utilidad, entonces a) toda expresión matemática, en algún momento de su existencia, ha carecido por completo de significado, a saber, antes de usarse; $b$ ) no es posible determinar a priori qué expresiones serán útiles y cuáles no, por lo que hay que adoptar un criterio diferente de significatividad - ¿podría sugerirse la consistencia sintáctica? Habrá que esperar aún casi doscientos años para obtener una respuesta ast.)

Mucho más podría decirse acerca de esto; lo que he presentado espero

26 Las citas que hasta ahora he presentado siguen poniendo en evidencia que los matemáticos no tenfan una idea clara de qué tipo de entidades [si lo eran] eran las entidades con las que trataban al ampliar sus sistemas numéricos. Los Kneale señalan esta situación en forma clara al decirnos: "Hasta la introducción de los números complejos, y éstos incluidos, el desarrollo fue inconsciente en el sentido de que los matemáticos extendieron su uso de la palabra 'número' sin darse cuenta exactamente de lo que estaban haciendo." [11], p. 398. 
que sea bastante para darle al lector una idea de los problemas que aquejaban a los matemáticos en la época de Berkeley; cuál era su naturaleza, qué temas se discutían y la manera como se llevaba esto a cabo. Dentro de este concierto de voces, la de Berkeley parece que surge como una voz consciente y lúcida junto a las expresiones casi o plenamente contradictorias que emitían los matemáticos sobre su materia. Sin embargo, y esto es de justicia decirlo, los matemáticos, sea lo que sea lo que pensaran de su propia labor, y de los frutos de ésta, siguieron puliendo y afinando sus herramientas hasta conseguir de las mismas resultados cada vez más finos y acertados. Pero, lograr esto fue posible, en gran medida, gracias al efecto que tuvieron mentes lúcidas y críticas como la de Berkeley que señalaron con agudeza los problemas de oscuridad, confusión e inexactitud que se encontraban en la base de la más "exacta" de todas las ciencias.

Para concluir, y con el deseo de presentar un cuadro más completo con relación al destino de los números imaginarios o complejos, traigo a colación una última cita acerca de estos números y un dato acerca de su representación final en términos geométricos.

La cita es del lógico y matemático inglés del siglo xix, Augustus de Morgan (1806-1871), quien aún en una fecha tan tardía como 1831, en su libro On the Study and Difficulties of Mathematics, llegó a decir lo siguiente:

Hemos mostrado que el símbolo $\sqrt{-a}$ carece de significado o, más bien, que es contradictorio de suyo y absurdo. Sin embargo, mediante tales símbolos se establece una parte del álgebra que es de gran utilidad. Esta depende del hecho, que debemos verificar experimentalmente, de que las reglas comunes del álgebra pueden aplicarse a estas expresiones [números complejos] sin que esto conduzca a ningún resultado falso. Un llamado así a la experiencia parece ir en contra de los principios establecidos al comienzo de esta obra. No podemos negar que, en realidad, esto es así, pero debemos recordar que ésta no es sino una parte pequeña y aislada de un tema inmenso al que estos principios se aplican en toda su extensión a todas sus otras ramas. ${ }^{27}$

Presento ahora el dato ofrecido: a finales del siglo xvirl, un agrimensor noruego, Caspar Wessel (1745-1818) escribe, en 1797, un ensayo sobre "La representación analítica de la dirección; un intento", que se publica en las memorias de 1799 de la Real Academia Danesa. En este ensayo, Wessel presenta una representación geométrica de los números

27 [9], p. 596. 
imaginarios en términos de vectores. La publicación, sin embargo, tuvo muy pocos lectores y llegó a ser conocida hasta 1897, año en que se traduce y se publica en francés.

En 1806, un tenedor de libros francés, Jean-Robert Argand (17681822), publica un pequeño libro, Essai sur une manière de représenter les quantités imaginaires dans les constructions géométriques. En esta publicación, Argand ofrece otro intento de representación de los números imaginarios en términos geométricos; esta representación difiere en algo de la de Wessel. La publicación de Argand tampoco tuvo influencia en el público matemático.

Finalmente, Carl Friedrich Gauss es el matemático que mayor influencia tiene para dejar perfectamente establecidos, en el ámbito de la matemática, a los números imaginarios o complejos. Desde 1799 los usa en sus varias demostraciones del teorema fundamental del álgebra ( $c f$. supra, pp. 166-167), pero han de pasar aún algunos años, hasta 1831, para que Gauss, de manera explícita y pública, describa la representación geométrica de los números imaginarios. Según Gauss, en la representación geométrica de los imaginarios (o complejos que, según señalamos, fue la denominación que él les dio), uno encuentra el "significado intuitivo de los números complejos completamente establecido y no se necesita más para admitir estas cantidades en el dominio de la aritmética".28

Tras un largo peregrinar entre incomprensión y burlas, los números imaginarios encontraron, al fin, su lugar dentro de la matemática. Sin embargo, debo recordarle al lector que su papel como elementos útiles de cálculo ya lo tenían, en 1731, dentro de la visión matemática general de Berkeley.

\section{BIBLIOGRAFIA}

[1] Berkeley, G.: Alciphron, or the Minute Philosopher (1732). Con una introducción de T. E. Jessop; en [4], III.

[2] —-: Philosophical Commentaries, generally called the Commonplace Book (1707-8). Una editio diplomatica transcrita y editada con una introducción y notas por A. A. Luce MC DD Litt D. Thomas Nelson and Sons Limited, Londres; 1944.

[3] —-: Siris (1744). Con prefacio, introducción y notas por T. E. Jessop; en [4], $V$, pp. 1-164.

[4] - The Works of George Berkeley Bishop of Cloyne; vols. I-IX. Edi-

28 Todos los datos que aquí he presentado los puede encontrar el lector en [9], pp. 631-2. 
tadas por A. A. Luce y T. E. Jessop. Thomas Nelson and Sons, Ltd., Londres; 1948-1957.

[5] Cajori, F.: A History of Mathematics (1893; 2a ed. 1919). Chelsea Publishing Company, Nueva York; 1980.

[6] Descartes, R.: The Geometry of Rene Descartes, with a facsimile of the first edition (1925). Traducción del latín y el francés de La Géométrie (1637), por David Eugene Smith y Marcia L. Latham. Dover Publications, Inc., Nueva York; 1954.

[7] Diderot, D. y D'Alembert, J.: Encyclopédie ou Dictionnaire Raisonné des Sciences, des Arts et des Metiers, par une Societé de Gens de Lettres. Nouvelle impression en facsimilé de la première edition de 1751-1780. Friedrich Frommann Verlag (Günther Holzboog); Stuttgart-Bad Cannstatt; 1966.

[8] Gamow, G.: One Two Three... Infinity; Facts and Speculations of Science (1947). Bantam Books, Nueva York; 1979.

[9] Kline, M.: Mathematical Thought from Ancient to Modern Times (1972). Oxford University Press, Nueva York; 1980 (6a impresión).

[10] —-: Mathematics, the Loss of Certainty (1980). Oxford University Press, Nueva York; 1981 (2a impresión).

[11] Kneale, W. \& M.: The Development of Logic. Oxford University Press, Oxford; 1962.

[12] Leibniz, G. W.: Gothofredi Guillelmi Leibnitii... Opera Omnia nunc primum collecta, in classes distributa, præfationibus \& indicibus exornata, studio Ludovici Dutens. Tomus Tertius, continens opera Mathematica. Genevæ, apud Fratres de Tournes. MDCCLXVIII.

[13] - - "Specimen Novum Analyseos, pro Scientia Infiniti, circa Summas \& Quadraturas"; publicado en el Acta Eruditorum de 1702. Recogido en [12], pp. 373-381.

[14] Péter, Rózsa: Playing with Infinity; Mathematical Explorations and Excursions. Traducción del húngaro (Játek a Végtelennel-Matematika Kivülállóknak, 1957) por el Dr. Z. P. Dienes. Dover Publications, Inc., Nueva York; 1961.

[15] Robles, José Antonio (comp.): Bertrand Russell: antologia I. SepDiana, México; 1982.

[16] Russell, B.: Our Knowledge of the External World as a Field for Scientific Method in Philosophy (1914). Georg Allen \& Unwin Ltd., Londres; 1961.

[17] Smith, D. E.: History of Mathematics vol. I: General Survey of the History of Elementary mathematics (1923).

vol. II: Special Topics of Elementary Mathematics (1925).

Dover Publications, Inc., Nueva York; 1958.

[18] Struik, D. J. (ed.): A Source Book in Mathematics, 1200-1800. Harvard University Press, Cambridge, Mass; 1969. 